\title{
Phenomenology of QCD threshold resummation for gluino pair production at NNLL
}

\section{Torsten Pfoh}

Deutsches Elektronensynchrotron DESY,

Platanenallee 6, D-15738 Zeuthen, Germany

E-mail: torsten.pfoh@desy.de

\begin{abstract}
We examine the impact of threshold resummation for the inclusive hadronic production cross section of gluino pairs at next-to-next-to-leading-logarithmic accuracy, compared to the exact next-to-leading-order cross section and the next-to-next-to-leadingorder approximation. Here, we apply formulas derived recently in the classical Mellin-space formalism. Moreover, we give the analytic input for the alternative momentum-space formalism and discuss the crucial points of the numeric implementation. We find that soft resummation keeps the hadronic cross section close to the fixed next-to-leading-order result.
\end{abstract}

KEYWORDS: Resummation, Supersymmetric Standard Model

ARXiv EPRINT: 1302.7202 


\section{Contents}

1 Introduction 1

2 Threshold resummation in Mellin space 3

3 Threshold resummation in momentum space 5

4 Implementation of the Mellin-space formalism 11

5 Implementation of the momentum-space formalism 13

6 Hadronic cross section $\quad 17$

$\begin{array}{llr}7 & \text { Conclusions } & 20\end{array}$

$\begin{array}{ll}\text { A Matching to the NNLO approximation } & 20\end{array}$

\section{Introduction}

Within the search for new physics at the LHC experiments, one hopes to find evidences for heavy color-charged particles. These appear in many scenarios for physics beyond the Standard Model (SM). A prominent candidate is the gluino which is a Majorana fermion and the superpartner of the SM gluon within various realizations of Supersymmetry (SUSY), as one of the most favorite models. Unfortunately, the direct search at ATLAS and CMS have only produced exclusion limits ${ }^{1}$ so far which, of course, depend on the model under consideration, and the assumptions on the SUSY particle spectrum.

In order to separate possible signals from the SM background, a crucial theoretical

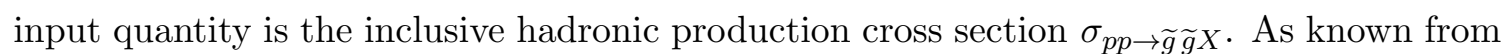
standard Quantum Chromodynamics (QCD), the latter is given by a sum over the various partonic production channels where the partonic cross sections $\hat{\sigma}_{i j \rightarrow \widetilde{g} \widetilde{g}}$ are convoluted with their respective parton luminosity functions $L_{i j}$. Explicitly, one has

$$
\begin{aligned}
\sigma_{p p \rightarrow \widetilde{g} \widetilde{g} X}\left(s, m_{\tilde{g}}^{2}, m_{\tilde{q}}^{2}, \mu_{f}^{2}, \mu_{r}^{2}\right) \\
\quad=\sum_{i, j=q, \bar{q}, g} \int_{4 m_{\tilde{g}}^{2} / s}^{1} d \tau L_{i j}\left(\tau, \mu_{f}^{2}\right) \hat{\sigma}_{i j \rightarrow \widetilde{g} \widetilde{g}}\left(\tau s, m_{\widetilde{g}}^{2}, m_{\widetilde{q}}^{2}, \mu_{f}^{2}, \mu_{r}^{2}\right),
\end{aligned}
$$

where the parton luminosities are themselves given by a convolution of the parton distribution functions (PDFs)

$$
L_{i j}\left(\tau, \mu_{f}^{2}\right)=\int_{0}^{1} d x_{1} \int_{0}^{1} d x_{2} \delta\left(x_{1} x_{2}-\tau\right) f_{i / p}\left(\mu_{f}^{2}, x_{1}\right) f_{j / p}\left(\mu_{f}^{2}, x_{2}\right)
$$

\footnotetext{
${ }^{1}$ See refs. $[1,2]$ for example.
} 
and we have introduced the hadronic center-of-mass (cms) energy $s$, the partonic cms energy $\hat{s}=\tau s$, the factorization scale $\mu_{f}$, and the renormalization scale $\mu_{r}$. The gluino mass is denoted by $m_{\widetilde{g}}$, and we assume mass degeneracy among the squarks flavors, therefore using a single scale $m_{\widetilde{q}}$.

At leading order (LO) in perturbation theory, gluino pair production is driven by the partonic sub processes of gluon fusion $g g \rightarrow \widetilde{g} \widetilde{g}$ and quark-antiquark annihilation $q \bar{q} \rightarrow \tilde{g} \widetilde{g}, q=d, u, s, c, b$. At next-to-leading-order (NLO), also the $g q$ channel opens [3]. Close to the threshold however, its contribution is suppressed compared to gluon fusion and quark-antiquark annihilation. The full NLO result has been implemented in the public program Prospino [4].

As very well known, the cross section develops powers of large logarithms $\ln (\beta)$ near the production threshold, where the velocity

$$
\beta=\sqrt{1-4 m_{\tilde{g}}^{2} / \hat{s}} \equiv \sqrt{1-\rho}
$$

of the produced particle pair goes to zero. The so-called threshold logarithms spoil the validity of the perturbative expansion in the strong coupling constant $\alpha_{s}$. However, they can be resummed systematically to all orders in perturbation theory, where the quantity $\alpha_{s} \ln (\beta)$ counts as order one. In this context, threshold logarithms are sometimes called soft logarithms and one talks about soft resummation. There are two approaches to soft resummation which we will shortly discuss below. We will refer to them as Mellin-space formalism $[5,6]$ and momentum-space formalism $[7,8]$.

Another difficulty arises through the exchange of soft gluons between the final state particles. At fixed order in perturbation theory, this gives rise to so-called Coulomb terms proportional to powers of $\alpha_{s} / \beta$. Obviously, with decreasing $\beta$, the above ratio becomes $\mathcal{O}(1)$ and should therefore be resummed as well. This can be done in the framework of nonrelativistic QCD (NRQCD). A joined soft and Coulomb resummation has been worked out in the momentum-space formalism [9]. In the Mellin-space formalism however, one would need to calculate the Mellin transformations of the NRQCD expressions analytically (or at least semi-analytically as a function of the Mellin moments), a problem which has not been solved so far. Therefore, Coulomb terms are included at fixed order only.

For any process, soft resummation up to so-called next-to-leading-logarithmic (NLL) accuracy requires the knowledge of the color-decomposed Born cross section with respect to the $\mathrm{SU}(3)_{c}$-color configuration of the produced (s)particle pair. At next-to-next-to-leadinglogarithmic (NNLL) precision, one needs the color decomposition of the NLO cross section near the threshold, where higher powers of $\beta$ are skipped. For gluino pair production, the state of the art in the momentum-space formalism is a combined soft and Coulomb resummation up to NLL accuracy [10]. Within the Mellin-space approach, the NLL results of ref. [11, 12] have been extended to NNLL precision recently in ref. [13]. Bound-state and finite-width effects at fixed order have been discussed in ref. $[14,15]$. In the context of NLL resummation, finite-width effects have been discussed recently in ref. [16]. It has been found that for $\Gamma / m_{\tilde{g}} \approx 5 \%$, the effect is of the order of the ambiguities due to higherorder contributions in the resummation formula. In this article, we work in the zero-width 
approximation which is very well justified if the squark and neutralino masses are about the order of the gluino mass. If one expands the NNLL threshold resummation formula up to $\alpha_{s}^{2}$ (modulo the factor $\alpha_{s}^{2}$ in the Born cross section), one reproduces the threshold logarithms up to next-to-next-to-leading-order (NNLO). One further obtains constant terms which are generically different at NNLO for the two approaches. However, a proper matching eliminates these constants and one is left with the threshold approximation $\left(\mathrm{NNLO}_{\mathrm{th}}\right)$, where, after factorization of the Born cross section, all NNLO constants are set to zero. At NLO, the constants are kept as these refer to the exact fixed order result.

If the gluino is heavy (of the order of $1 \mathrm{TeV}$ ), the threshold-enhanced terms give the dominant contribution at given order in perturbation theory. Combining the full NLO result with the $\mathcal{O}\left(\alpha_{s}^{2}\right)$ threshold enhanced terms to the NNLO approximation ( $\mathrm{NNLO}_{\text {approx }}$ ), one finds an increase of the cross section of about $20 \%$ with respect to the fixed NLO result [13]. The question of interest is how these findings change in the presence of NNLL resummation. Although the resummation formula for soft logarithms has been derived analytical, it has not been implemented yet. In this context, a general question is how to deal with ambiguities related to the choice of scales or the treatment of the Landau pole in momentum integrals. A different source of uncertainty comes from the unknown squark masses. However, the hadronic process is mainly driven by gluon fusion which has only a weak dependence on $m_{\widetilde{q}}$. As a consequence, the impact of the squark masses on the cross section is negligible to good approximation. In the threshold limit, the dependence on $m_{\widetilde{q}}$ actually vanishes. The impact of higher order contributions is estimated by a variation of the factorization and renormalization scale. As discussed in ref. [13], the main source of uncertainty comes from the shape of the gluon PDFs at high momentum fraction $x$, and the value of $\alpha_{s}\left(M_{Z}\right)$. The possible large discrepancies are not covered by the individual PDF errors, and give the main uncertainty when setting a reliable exclusion limit.

In this article, we study the impact of NNLL soft resummation in the Mellin-space approach. In the sections 2 and 3, we briefly review the methods for soft (and Coulomb) resummation in the Mellin- and momentum-space formalism. Concerning the latter, we derive the color-decomposed hard function at NLO which is needed for NNLL resummation. We discuss the ingredients of the potential function which collects all results from NRQCD derived from the NLO non-relativistic potential. In the sections 4 and 5, we discuss technical aspects of the numerical implementation of the resummation formulas in the two approaches, and explicitly apply the Mellin-space formalism. The inclusive hadronic cross section is discussed in section 6 and we give our conclusion in section 7. An explicit formula for the matching of the momentum-space resummation formula onto the NNLO approximation is provided in the appendix.

\section{Threshold resummation in Mellin space}

The traditional approach to threshold resummation has been invented in ref. [5, 6], see also ref. [17-22] for further discussions. Resummation is performed in Mellin space after introducing moments $N$ with respect to the variable $\rho=4 m_{\widetilde{g}}^{2} / \hat{s}$ of momentum space. 
Neglecting the dependencies on $\mu_{f}$ and $\mu_{r}$, one has

$$
\hat{\sigma}_{i j}^{N}\left(m_{\tilde{g}}^{2}\right)=\mathbf{M}\left[\hat{\sigma}_{i j}\left(m_{\widetilde{g}}^{2}\right)\right](N)=\int_{0}^{1} d \rho \rho^{N-1} \hat{\sigma}_{i j}\left(\hat{s}, m_{\widetilde{g}}^{2}\right),
$$

where the threshold limit $\beta \rightarrow 0$ corresponds to $N \rightarrow \infty$. The resummation of threshold logarithms is achieved by the formula

$$
\hat{\sigma}_{i j, \mathbf{I}}^{\mathrm{res}, \mathrm{N}}=\hat{\sigma}_{i j, \mathbf{I}}^{B, N} g_{i j, \mathbf{I}}^{0} g_{i j, \mathbf{I}}^{0 C}(N+1) \exp \left[G_{i j, \mathbf{I}}(N+1)\right]+\mathcal{O}\left(N^{-1} \ln ^{n} N\right) .
$$

All contributions from soft and collinear radiation exponentiate and are collected in the function $G_{i j, \mathbf{I}}$. To NNLL accuracy, it may conveniently be expanded according to

$$
G_{i j, \mathbf{I}}(N)=\ln (\widetilde{N}) \cdot g_{i j}^{1}(\widetilde{\lambda})+g_{i j, \mathbf{I}}^{2}(\widetilde{\lambda})+a_{s} g_{i j, \mathbf{I}}^{3}(\widetilde{\lambda})+\ldots,
$$

where $\widetilde{N} \equiv N \exp \left(\gamma_{E}\right)$ with $\gamma_{E}$ denoting the Euler-Mascheroni constant, $\widetilde{\lambda} \equiv a_{s} \beta_{0} \ln \tilde{N}$, and $a_{s} \equiv \alpha_{s} /(4 \pi)$. The explicit expressions are calculated by a double integral over a set of anomalous-dimension functions. Some of these depend on the $\mathrm{SU}(3)_{c}$-color configuration of the final-state gluino pair, which we label by a capital index I. The color-summed partonic cross section for a given channel is simply given by

$$
\hat{\sigma}_{i j \rightarrow \widetilde{g} \widetilde{g}}=\sum_{\mathbf{I}} \hat{\sigma}_{i j, \mathbf{I}} .
$$

The possible final-state color configurations are obtained by a decomposition of the initial color states into irreducible representations according to

$$
\begin{aligned}
& \mathbf{8} \times \mathbf{8}=\mathbf{1}_{s}+\mathbf{8}_{s}+\mathbf{8}_{a}+\mathbf{1 0}+\overline{\mathbf{1 0}}+\mathbf{2 7 _ { s }}, \\
& \mathbf{3} \times \overline{\mathbf{3}}=\mathbf{1}_{s}+\mathbf{8}_{s}+\mathbf{8}_{a}
\end{aligned}
$$

for gluon fusion and quark-antiquark annihilation, respectively. There are symmetric $(s)$ and anti-symmetric $(a)$ color states. The corresponding quadratic Casimir operators $C_{\mathbf{I}}$, which show up in the color-decomposed partonic cross section, take the values

$$
C_{\mathbf{I}}=\{0,3,6,8\} \quad \text { for } \quad \mathbf{I}=\{\mathbf{1}, \mathbf{8}, \mathbf{1 0}, \mathbf{2 7}\}
$$

independent from the symmetry properties. The (color-decomposed) Born cross section $\hat{\sigma}_{i j, \mathbf{I}}^{B}$ factors out in eq. (2.2) which is also true for the fixed order partonic cross section in the threshold limit $\hat{\sigma}_{i j, \mathbf{I}}^{\text {th }}$. The matching constant $g_{i j, \mathbf{I}}^{0}$ collects all contributions independent of $N$ and has to be determined order by order in perturbation theory by expanding ${ }^{2}$ the right-hand side of eq. (2.2) in $\alpha_{s}$ and matching onto $\hat{\sigma}_{i j, \mathbf{I}}^{\text {th }}$. Coulomb corrections are not resummed by the above ansatz. They are formally treated as part of the hard scattering function on which the exponent has to be matched. Therefore, one introduces a second matching term $g_{i j, \mathbf{I}}^{0 C}$ [23] as a power series in $\alpha_{s}$ which is actually a function of $N$. If Coulomb corrections are not considered, $g_{i j, \mathbf{I}}^{0 C}$ reduces to a factor one.

\footnotetext{
${ }^{2}$ This produces all the threshold logarithms of the form $\ln ^{k}(N)$.
} 
Note that starting from NNLO, one has non-relativistic corrections of non-Coulomb type which cause the cross section to depend on the spin-configuration $S$ of the produced heavy particle pair [24-28]. These terms are also not treated by the exponent (2.3) and thus are fully hosted by the hard matching constant. As they also depend on the Mellin variable $N$, it is convenient to include them into $g_{i j, \mathbf{I}}^{0 C}(N)$, although they do not arise from Coulomb exchange. Alternatively, one could also introduce a third matching constant $g_{i j, \mathbf{I}}^{0 N C}(N)$. The spin dependence is described by a parameter $v_{\text {spin }}$ which is zero for the gluino pair being in a spin singlet $(S=0)$ configuration and $-2 / 3$ for a spin triplet $(S=1)$, see ref. [24]. At the threshold, gluon fusion gives rise to a singlet, where quark-antiquark annihilation produces a triplet. All ingredients of the NNLL threshold resummation in Mellin space for gluino pair production can be found in ref. [13].

\section{Threshold resummation in momentum space}

An alternative approach which features resummation in momentum space has been invented in ref. $[7,8]$ in the context of deep inelastic scattering. Here, soft and collinear radiation are described in the framework of soft-collinear effective theory. In ref. [9], this method has been adopted to heavy (s)particle pair production and extended to the summation of Coulomb corrections. The starting point is the observation that near the threshold the partonic production cross section can be factorized into a hard function $H$, a soft function $W$ which collects soft fluctuations, and a potential function $J$ which sums Coulomb exchange. Below the threshold, one has a discrete spectrum of bound-states. If these are taken into account, $J$ should be expressed as a function of the energy $E=\sqrt{\hat{s}}-2 m_{\widetilde{g}}$ relative to the threshold $[9,10,29]$.

At fixed order in $\alpha_{s}$, the partonic cross section close to the threshold can be written as $[9]$

$$
\hat{\sigma}_{i j \rightarrow \widetilde{g} \widetilde{g}}(\hat{s}, \mu)=\sum_{S} \sum_{\mathbf{I}} H_{i j, \mathbf{I}}^{S}(\mu) \int_{0}^{\infty} d \omega J_{\mathbf{I}}^{S}\left(E-\frac{\omega}{2}\right) W_{\mathbf{I}}(\omega, \mu)
$$

where one sums over the spin and color configurations of the final-state particle pair. The spin dependence enters the hard and the potential function at NNLO first. In order to account for the different scales of the problem and to achieve resummation of threshold logarithms, one calculates the hard function at the hard scale $\mu_{h}$ and the soft function at soft scale $\mu_{s}$. Then, one solves renormalization group equations for the hard and the soft function which in the latter case, sums the soft logarithms. The solutions are used to evolve $H\left(\mu_{h}\right)$ and $W\left(\mu_{s}\right)$ to the common scale $\mu_{f}$ which is used for the convolution of the partonic cross sections with the parton luminosities. The general all order resummation formula reads [9]

$$
\begin{aligned}
\hat{\sigma}_{i j \rightarrow \widetilde{g} \widetilde{g}}^{\mathrm{res}}\left(\hat{s}, \mu_{f}\right)= & \sum_{S} \sum_{\mathbf{I}} H_{i j, \mathbf{I}}^{S}\left(\mu_{h}\right) U_{i j, \mathbf{I}}\left(m_{\widetilde{g}}, \mu_{h}, \mu_{s}, \mu_{f}\right) \\
& \times \int_{0}^{\infty} d \omega \frac{J_{\mathbf{I}}^{S}\left(E-\frac{\omega}{2}\right)}{\omega}\left(\frac{\omega}{2 m_{\widetilde{g}}}\right)^{2 \eta} \tilde{s}_{i j, \mathbf{I}}\left(2 \ln \left(\frac{\omega}{\mu_{s}}\right)+\partial_{\eta}, \mu_{s}\right) \frac{e^{-2 \gamma_{E} \eta}}{\Gamma(2 \eta)}
\end{aligned}
$$


where the summation of Sudakov-double logarithms is included in the evolution function $U_{i j, \mathbf{I}}$ which is given by

$$
\begin{aligned}
U_{i j, \mathbf{I}}\left(m_{\widetilde{g}}, \mu_{h}, \mu_{s}, \mu_{f}\right)= & \left(\frac{4 m_{\widetilde{g}}^{2}}{\mu_{h}^{2}}\right)^{-2 a_{\Gamma}\left(\mu_{h}, \mu_{s}\right)}\left(\frac{\mu_{h}^{2}}{\mu_{s}^{2}}\right)^{\eta} \times \exp \left[4\left(S\left(\mu_{h}, \mu_{f}\right)-S\left(\mu_{s}, \mu_{f}\right)\right)\right. \\
& \left.-2 a_{i j, \mathbf{I}}^{V}\left(\mu_{h}, \mu_{s}\right)+2 a_{i}^{\phi}\left(\mu_{s}, \mu_{f}\right)+2 a_{j}^{\phi}\left(\mu_{s}, \mu_{f}\right)\right] .
\end{aligned}
$$

The function $\tilde{s}_{i j, \mathbf{I}}\left(\rho, \mu_{s}\right)$ is the Laplace transform of the $\overline{\mathrm{MS}}$-renormalized soft function $\bar{W}_{\mathbf{I}}(\omega, \mu)$ which, to NLO accuracy, is given by

$$
\tilde{s}_{i j, \mathbf{I}}(\rho, \mu)=1+a_{s}(\mu)\left[\left(C_{i}+C_{j}\right)\left(\rho^{2}+\frac{\pi^{2}}{6}\right)-2 C_{\mathbf{I}}(\rho-2)\right]+\mathcal{O}\left(a_{s}^{2}\right)
$$

where we again define $a_{s} \equiv \alpha_{s} /(4 \pi)$. The auxiliary variable $\eta$ in the eq. (3.2) is set to $\eta=2 a_{\Gamma}\left(\mu_{s}, \mu_{f}\right)$ after performing the derivative. It contains single logarithms [10] which can be seen by expanding $\eta=4 a_{s}\left(C_{i}+C_{j}\right) \ln \left(\mu_{s} / \mu_{f}\right)+\mathcal{O}\left(a_{s}^{2}\right)$. From the latter expression one also learns that $\eta$ is negative and tends towards zero as $\mu_{s}$ approaches $\mu_{f}$. This behavior is preserved at higher orders in $\alpha_{s}$. As a consequence, the integration kernel $\left(\omega /\left(2 m_{\tilde{g}}\right)\right)^{2 \eta} / \omega$ has to be understood in a distributional sense [8] as will be further discussed in section 5 .

The quadratic Casimir operators $C_{i}$ and $C_{\mathbf{I}}$ depend on the color configuration of the initial and final states, respectively. For gluino pair production via gluon fusion we have $C_{i}=C_{j}=C_{A}=3$, for quark-antiquark annihilation we have $C_{i}=C_{j}=C_{F}=4 / 3$. With $\mu_{s} \leq \mu_{f}$ it hence follows that $\eta \leq 0$. The function $a_{\Gamma}(\nu, \mu)$ and the Sudakov exponent $S(\nu, \mu)$ are given to NNLO precision in eq. (86) and eq. (87) of ref. [30]. For NNLL resummation, the respective NLO expressions are sufficient. For $i=j$, these are functions of expansion coefficients $\Gamma_{k}^{i}$ of the cusp anomalous dimension

$$
\Gamma_{\text {cusp }}^{i}=\sum_{k=0}^{\infty} \Gamma_{k}^{i} a_{s}^{k+1} \equiv C_{i} \gamma_{\text {cusp }}
$$

and coefficients of the QCD beta-function $\beta_{k}$. The latter are listed in eq. (85) of the above article, whereas the cusp anomalous dimension is given for the quark-antiquark channel in eq. (81). For gluon fusion, one just has to multiply these results by $C_{A} / C_{F}$ according to the Casimir scaling indicated in eq. (3.5) above. For $i \neq j$, one would replace

$$
\Gamma_{\text {cusp }}^{i} \rightarrow \Gamma_{\text {cusp }}^{i j}=\frac{1}{2}\left(\Gamma_{\text {cusp }}^{i}+\Gamma_{\text {cusp }}^{j}\right) \equiv \frac{1}{2}\left(C_{i}+C_{j}\right) \sum_{k=0}^{\infty} \gamma_{\text {cusp }}^{(k)} a_{s}^{k+1} .
$$

Note also that the coefficients $\Gamma_{k}^{i}$ coincide with the coefficients $A_{i}^{(l)}$ with $l=k+1$, used in the Mellin-space formalism (see ref. [13,31]). The functions $a_{i j, \mathbf{I}}^{V}$ and $a_{i}^{\phi}$ in eq. (3.3) are obtained in analogy to $a_{\Gamma}$ by a replacement of $\Gamma_{\text {cusp }}^{i j}$ with $\gamma_{i j, \mathbf{I}}^{V}=\gamma_{i}+\gamma_{j}+\gamma_{H, \mathbf{I}}$ in the first case, and with $\left(\gamma_{i}^{\phi}+\gamma_{j}^{\phi}\right) / 2$ in the second. The single particle anomalous dimension functions $\gamma_{i}$ are related to soft radiation from the (mass-less) initial-state particles and are 
collected in appendix A of ref. [32]. The anomalous dimension $\gamma_{H, \mathbf{I}}$ refers to soft radiation connected to a massive final-state particle in the color representation I. NLO results are given in eqs. (3.31) (3.32) of ref. [33]. Here, one can also find expansion coefficients of $\gamma_{i}^{\phi}$ which are related to the evolution of the parton distribution function. Explicit expressions up to NLO for color-triplets and octets are given in eqs. (D.17)-(D.20).

When taking results from the literature, care has to be taken with respect to the meaning of the parameter $n_{f}$. Typically, $n_{f}$ denotes the number of active flavors which contribute to the evolution of $\alpha_{s}$. So it does in the articles listed above where the various coefficients of the QCD beta function and anomalous dimensions are collected. However, in some papers about gluino pair production at the threshold ${ }^{3} n_{f}$ denotes the total amount of quark (and squark) flavors which, depending on the renormalization scheme, may differ from the number of active flavors.

As discussed in ref. [9], the all-order resummation formula (3.2) does not depend on the hard and soft scale $\mu_{h}$ and $\mu_{s}$. However, as all ingredients are only known to finite order in $\alpha_{s}$, a truncation of the perturbative expansion induces a residual dependence on these scales. A good perturbative behavior is obtained by a suitable choice for $\mu_{h}$ and $\mu_{s}$. For the case of gluino pair production, these are $\mu_{h} \approx k_{h} m_{\widetilde{g}}$ and $\mu_{s}=k_{s} m_{\widetilde{g}} \beta^{2}$ with $k_{h}$ and $k_{s}$ being numbers of $\mathcal{O}(1)$. The choice of a running soft scale $\sim m_{\widetilde{g}} \beta^{2}$ is also necessary to reproduce the threshold logarithms at NNLO (fixed order) [29]. In practice, the above choice becomes problematic when $\beta$ tends zero. Moreover, $\alpha_{s}\left(\mu_{s}\right)$ approaches the Landau pole. Therefore, one should introduce a parameter $\beta_{\text {cut }}[29]$. For $\beta>\beta_{\text {cut }}$, a running soft scale is used such that the perturbative expansion is not spoiled by large logarithms. On the other hand, for $\beta<\beta_{\text {cut }}$, the scale is fixed and kept in the perturbative regime. Note that a special treatment of the Landau pole is also required in the Mellinspace approach when performing the Mellin inversion (see refs. [19, 20] for instance). In the momentum-space approach a proper choice of $\beta_{\text {cut }}$ should keep the ambiguities which arise when matching the resummed to the fixed order cross section as small as possible. For gluino pair production, this has been analyzed in ref. [10]. Finally, we note that the choice of the soft scale actually determines what is being resummed. A dedicated discussion can be found in ref. [34]. Within the Mellin-space formalism on the other hand, one has the implicit scale choices $\mu_{s}=m_{\widetilde{g}} / N$ and $\mu_{h}=\mu_{f}$, and the two approaches are formally identical up to $\mathcal{O}(1 / N)$-terms (see for instance the discussion in ref. [30]).

Coming back to the momentum-space approach, one requires the potential function $J_{\mathbf{I}}^{S}(E)$ which describes the exchange of soft gluons between the final-state particles as well as higher order corrections coupled to these diagrams. At fixed order in perturbation theory, pure Coulomb corrections (which correspond to ladder diagrams) give rise to terms proportional to $\left(\alpha_{s} / \beta\right)^{n}$. As already mentioned, they can be treated by methods of NRQCD using the LO non-relativistic potential and have been found to be summed by a Sommerfeld factor $\Delta^{C}$ [35]. Starting from NLO, one also has to deal with terms multiplied by $\alpha_{s}\left(\alpha_{s} / \beta\right)^{n}$. Their summation requires the non-relativistic potential at NLO $[24,26]$. The LO and NLO Greens functions of the non-relativistic Schrödinger equation, $G_{C, \mathbf{I}}^{(0)}(E)$

\footnotetext{
${ }^{3}$ See ref. $[13,15]$ for instance.
} 
and $G_{C, \mathbf{I}}^{(1)}(E)$, are given in ref. [9, 29], and the contributions to the potential function are obtained by taking two times the imaginary parts. For positive values of the energy $E$ relative to the threshold, one finds the simple leading expression

$$
\begin{aligned}
J_{\mathbf{I}}^{(0)}(E) & =\frac{m_{\widetilde{g}}^{2}}{2 \pi} \sqrt{\frac{E}{m_{\widetilde{g}}}} \Delta^{C}\left(\pi \alpha_{s} \sqrt{\frac{m_{\widetilde{g}}}{E}} D_{\mathbf{I}}\right) \theta(E) \\
& =\frac{m_{\widetilde{g}}^{2}}{2 \pi} \sqrt{\frac{E}{m_{\widetilde{g}}}}\left[1-a_{s} 2 \pi^{2} D_{\mathbf{I}} \sqrt{\frac{m_{\widetilde{g}}}{E}}+a_{s}^{2} \frac{4 \pi^{4} D_{\mathbf{I}}^{2}}{3} \frac{m_{\widetilde{g}}}{E}+\mathcal{O}\left(a_{s}^{3}\right)\right] \theta(E),
\end{aligned}
$$

where the Sommerfeld factor is given by $\Delta^{C}(x)=x /(\exp (x)-1)$, and we have introduced the color coefficient $D_{\mathbf{I}}$ of the QCD potential

$$
D_{\mathbf{I}}=\frac{1}{2} C_{\mathbf{I}}-C_{A}=\{-3,-3 / 2,0,1\} \quad \text { for } \quad \mathbf{I}=\{\mathbf{1}, \mathbf{8}, \mathbf{1 0}, \mathbf{2 7}\} .
$$

Note that close to the threshold where $E \ll 2 m_{\tilde{g}}$, one has $\sqrt{m_{\tilde{g}} / E}=1 / \beta+\mathcal{O}(\beta)$ with $\beta \ll 1$. This gives rise to the expression $\Delta^{C}\left(\pi \alpha_{s} D_{\mathbf{I}} / \beta\right)$ also described in ref. [11, 13] which makes the summation of $1 / \beta$-terms evident. For $D_{\mathbf{I}}<0$ the potential is attractive, for $D_{\mathbf{I}}>0$ it is repulsive.

The NLO Coulomb function $J_{\mathbf{I}}^{(1)}(E)$ can not be brought to a simple form. However, it can be expressed as a linear combination of dilogarithms and nested harmonic sums with a complex argument $\lambda(E)=\alpha_{s}\left(-D_{\mathbf{I}}\right) /\left(2 \sqrt{-E / m_{\tilde{g}}}\right)$ [29]. As a first approximation, it may be sufficient to include $J_{\mathbf{I}}^{(1)}$ at fixed order in $\alpha_{s}$. It reads

$$
J_{\mathbf{I}}^{(1)}(E)=\frac{m_{\widetilde{g}}^{2}}{2 \pi} a_{s}^{2} 2 \pi^{2} D_{\mathbf{I}}\left[\beta_{0}\left(\ln \left(\frac{E}{m_{\widetilde{g}}}\right)+2 \ln \left(\frac{2 m_{\widetilde{g}}}{\mu_{C}}\right)\right)-a_{1}\right]+\mathcal{O}\left(a_{s}^{3}\right)
$$

with $a_{1}=31 / 9 C_{A}-10 / 9 n_{l}$. The choice of the scale $\mu_{C}$ is discussed in detail in ref. [29]. The complete fixed order non-relativistic corrections at NNLO are obtained by adding to eq. (3.9) the convolution of the $\mathcal{O}\left(\alpha_{s}\right)$-Sommerfeld term with the NLO threshold approximation (without the Coulomb term), as well as a non-Coulomb contribution discussed below. The full result has been given in ref. [24] first.

If the potential is attractive, discrete bound states may develop below the threshold. Ignoring the gluino widths, these are described by

$$
J_{\mathbf{I}}^{\text {bound }}(E)=2 \sum_{n=1}^{\infty} \delta\left(E-E_{n}\right)\left(\frac{m_{\widetilde{g}} \alpha_{s}\left(-D_{\mathbf{I}}\right)}{2 n}\right)^{3}\left(1+\frac{\alpha_{s}}{4 \pi} \delta r_{1}\right) \theta\left(-D_{\mathbf{I}}\right), \quad(E<0),
$$

for S-wave production [9, 29], where the bound-state energies are given by

$$
E_{n}=-\frac{m_{\widetilde{g}} \alpha_{s}^{2} D_{\mathbf{I}}^{2}}{4 n^{2}}\left(1+\frac{\alpha_{s}}{4 \pi} e_{1}\right)
$$

The correction terms $\delta r_{1}$ and $e_{1}$ stem from the NLO Coulomb Greens function [36] and can be found in ref. [29], eqs. (B.4) and (B.5). We stress that bound-state production $(2 \rightarrow 1$ process) has different kinematics opposed to pair production $(2 \rightarrow 2$ process $)$ and therefore 
requires different fixed order formulas already at the Born level. The NLO cross section for gluinonium production has been studied in ref. [15].

As mentioned above, the non-relativistic potential also exhibits spin-dependent terms of non-Coulomb type starting from NNLO. Due to explicit results for the threshold enhanced part of the NNLO cross section [13], we know that these terms play a minor role for gluino pair production. They comply with around $5 \%$ of the total NNLO contribution to the partonic cross section. Thus, it is seems to be sufficient to include these terms at fixed order which has also been done for top-quark pair production [29]. We follow the latter reference and define the factor

$$
\Delta_{\mathrm{NC}, \mathbf{I}}^{S}(E)=D_{\mathbf{I}}\left(C_{A}-2 D_{\mathbf{I}}\left(1+v_{\text {spin }}\right)\right) \theta(E),
$$

where the parameter $v_{\text {spin }}$ has been defined above. In summary, the potential function required for NNLL resummation is given by

$$
J_{\mathbf{I}}^{S}(E)=J_{\mathbf{I}}^{(0)}(E)\left(1+\alpha_{s}^{2} \ln (\beta) \Delta_{\mathrm{NC}, \mathbf{I}}^{S}(E)\right)+J_{\mathbf{I}}^{(1)}(E),
$$

where $\ln (\beta) \approx \ln \left(\sqrt{E / m_{\tilde{g}}}\right)$.

As last ingredient of the NNLL resummation formula, the hard function $H_{i j, \mathbf{I}}^{S}\left(\mu_{h}\right)$ is needed at NLO. At LO, it is related to the partonic Born cross section [9, 10, 29]

$$
\hat{\sigma}_{i j, \mathbf{I}}^{(0)}\left(\mu_{h}\right) \approx \frac{m_{\widetilde{g}}^{2}}{2 \pi} \sqrt{\frac{E}{m_{\widetilde{g}}}} H_{i j, \mathbf{I}}^{(0)}\left(\mu_{h}\right) \approx \frac{m_{\widetilde{g}}^{2}}{2 \pi} \beta H_{i j, \mathbf{I}}^{(0)}\left(\mu_{h}\right),
$$

where the approximations are valid in the limit $\hat{s} \rightarrow 4 m_{\tilde{g}}^{2}$. Near the threshold, the LO cross section is factored out from higher order contributions, where, apart from an overall factor $\beta$ due to the Born term, all positive powers of $\beta$ are set to zero. Writing

$$
H_{i j, \mathbf{I}}\left(\mu_{h}\right)=H_{i j, \mathbf{I}}^{(0)}\left(\mu_{h}\right)\left[1+a_{s}\left(\mu_{h}\right) H_{i j, \mathbf{I}}^{(1)}\left(\mu_{h}\right)+a_{s}^{2}\left(\mu_{h}\right) H_{i j, \mathbf{I}}^{(2)}\left(\mu_{h}\right)+\mathcal{O}\left(a_{s}^{3}\right)\right],
$$

the required NLO hard functions are obtained by comparing the NLO fixed order threshold results with the NLO expansion of eq. (3.2). For this purpose, it is sufficient to set $J_{\mathbf{I}}^{S}=J_{\mathbf{I}}^{(0)}$, and we require the various anomalous dimension functions at LO only. Using $\gamma_{\text {cusp }}^{(0)}=4, \gamma_{H, \mathbf{I}}^{(0)}=-2 C_{\mathbf{I}}, \gamma_{i}^{\phi(0)}=-\gamma_{i}^{(0)}$, and $E=m_{\widetilde{g}} \beta^{2}$, we find in agreement with eq. (D.3) from ref. [9]

$$
\begin{aligned}
\hat{\sigma}_{i j \rightarrow \tilde{g} \widetilde{g}}^{\mathrm{res}, \exp }\left(\hat{s}, \mu_{f}\right)= & \sum_{\mathbf{I}} \frac{m_{\tilde{g}}^{2}}{2 \pi} \beta H_{i j, \mathbf{I}}\left(\mu_{h}\right)\left[1+a_{s}\left(-\frac{2 D_{\mathbf{I}} \pi^{2}}{\beta}+4\left(C_{i}+C_{j}\right) \ln ^{2}\left(8 \beta^{2}\right)\right.\right. \\
& -4\left(C_{\mathbf{I}}+\left(C_{i}+C_{j}\right)\left(4+L_{f \tilde{g}}\right)\right) \ln \left(8 \beta^{2}\right)+\left(C_{i}+C_{j}\right) L_{f \tilde{g}}^{2} \\
& +2\left(C_{\mathbf{I}}+\left(C_{i}+C_{j}\right)\left(4-L_{f h}\right)\right) L_{f \tilde{g}}+12 C_{\mathbf{I}}+\left(C_{i}+C_{j}\right)\left(32-\frac{11}{6} \pi^{2}\right) \\
& \left.\left.-\left(2 C_{\mathbf{I}}-4\left(C_{i}+C_{j}\right) \ln (2)-\left(\gamma_{i}^{(0)}+\gamma_{j}^{(0)}\right)\right) L_{f h}+\left(C_{i}+C_{j}\right) L_{f h}^{2}\right)+\mathcal{O}\left(a_{s}^{2}\right)\right],
\end{aligned}
$$


where we have defined $L_{f h}=\ln \left(\mu_{f}^{2} / \mu_{h}^{2}\right)$, and $L_{f \tilde{g}}=\ln \left(\mu_{f}^{2} / m_{\widetilde{g}}^{2}\right)$. The dependence on the soft scale has canceled between the soft function and the expansion of the evolution function. The terms proportional to $L_{f h}$ run the hard function $H_{i j, \mathbf{I}}^{(1)}\left(\mu_{h}\right)$ down to the factorization scale $\mu_{f}$, see also the discussion in ref. [9].

The explicit evaluation of the soft and the potential function at NLO has produced all threshold enhanced terms proportional to $\ln ^{k}\left(8 \beta^{2}\right)(k=1,2)$ and $1 / \beta$ which are easily identified within the fixed order cross section in the threshold limit, given in the refs. [3] and [13]. However, the renormalization scale has been set equal to the factorization scale in the latter articles. Adopting this choice simplifies the right-hand side of eq. (3.16) due to $L_{f h}=0$. From now on, we set $\mu_{h}=\mu_{f}=\mu, \alpha_{s}(\mu) \equiv \alpha_{s}$, and switch to the notation of ref. [13], where $L_{f \tilde{g}} \equiv L_{\mu}$. The NLO hard functions for gluino pair production in the momentum-space formalism then read

$$
\begin{aligned}
H_{g g, \mathbf{I}}^{(1)}(\mu)= & 4 C_{1, \mathbf{I}}^{g g}-12 C_{\mathbf{I}}-C_{A}\left(64-\frac{11 \pi^{2}}{3}\right) \\
& -\left(2 C_{\mathbf{I}}-8 C_{A} \ln (2)\right) L_{\mu}-2 C_{A} L_{\mu}^{2} \\
H_{q \bar{q}, \mathbf{8}_{a}}^{(1)}(\mu)= & 4 C_{1, \boldsymbol{8}_{a}}^{q \bar{q}}-12 C_{A}-C_{F}\left(64-\frac{11 \pi^{2}}{3}\right) \\
& -\left(2 C_{A}-8 C_{F} \ln (2)-2 \beta_{0}+6 C_{F}\right) L_{\mu}-2 C_{F} L_{\mu}^{2}
\end{aligned}
$$

in the $\overline{\mathrm{MS}}$ scheme with $n_{l}=5$ active light (mass-less) quark flavors. In the gluon fusion channel, only color-symmetric parts contribute near the threshold. Therefore, one has to sum over $\mathbf{I}=\mathbf{1}, \mathbf{8}, \mathbf{2 7}$. On the other hand, in the quark-antiquark annihilation channel, one only has contributions from the anti-symmetric octet to first approximation. The oneloop matching constants $C_{1, \mathbf{I}}^{g g}$ and $C_{1, \mathbf{8}_{a}}^{q \bar{q}}$ are given in eqs. (39) and (40) of ref. [13]. They are functions of the ratio $r=m_{\tilde{q}}^{2} / m_{\tilde{g}}^{2}$ of the squared squark and gluino masses.

If one expands the resummation formula (3.2) up to $\mathcal{O}\left(\alpha_{s}^{2}\right)$, one obtains some constants besides the threshold logarithms at NNLO. In order to obtain the full NNLO hard coefficients $H_{i j, \mathbf{I}}^{(2)}$, a dedicated two-loop calculation is necessary to determine all constant terms. As these are not available at the moment, it is common to keep only threshold enhanced terms. Thus, if one wishes to match the NNLL resummation onto the NNLO approximation, one requires a cancellation of all constants in the resummation formulas at $\mathcal{O}\left(\alpha_{s}^{2}\right)$. Within the Mellin-space approach, this is achieved by a proper choice of $g_{i j, \mathbf{I}}^{0}$ in eq. (2.2), see ref. [13]. For an analogous treatment in the momentum-space approach, we need to compute the next order in eq. (3.16). Keeping only the $\mathcal{O}\left(a_{s}^{0}\right)$ contribution of the potential function, $m_{\tilde{g}}^{2} \beta /(2 \pi)$, and the hard function up to $\mathcal{O}\left(a_{s}\right)$, the convolution with the NLO soft function and multiplication with the evolution function reproduces the soft logarithms $\ln ^{n}(\beta)(n=1, \ldots, 4)$ and their coefficients at NNLO if, and only if, we choose $\mu_{s}=k_{s} m_{\widetilde{g}} \beta^{2}$. The explicit fixed-order expressions can be found in ref. [13, 24]. The NNLO coefficient of the hard function has to be chosen such that the constants of the above expansion cancel at $\mathcal{O}\left(\alpha_{s}^{2}\right)$. Here, one should keep in mind that also the yet unknown NNLO soft function, if taken into account, produces constant pieces at $\mathcal{O}\left(a_{s}^{2}\right)$. In 
our case, we just arrange the NNLO hard function in such a way that all NNLO constant pieces cancel. This ensures a proper matching on the approximated NNLO cross section under usage of the NLO soft function. The explicit result is given in appendix A. The relative impact on the total hard function is rather small, typically at the percent level. Finally, we mention that for NNLL+NNLO approx accuracy, one can also set $H_{i j, \mathbf{I}}^{(2)}=0$, if the matching to the full fixed order (see discussion in the next section) is performed at the NNLO level. This differs from the choice in eq. (A.1) only in higher order terms beyond NNLL accuracy, and is applied in ref. [29]. For NNLL resummation, all input functions to the resummation formula are needed at $\mathcal{O}\left(\alpha_{s}\right)$. Note that the NLO expression of the Sudakov exponent $S(\nu, \mu)$ requires the coefficients of the cusp anomalous dimension and the QCD beta function up to NNLO (see ref. [30]). All other anomalous dimension functions are required at $\mathrm{NLO}$ only.

\section{Implementation of the Mellin-space formalism}

In this section, we discuss the numerical implementation of the resummation formula (2.2). The following procedure is required in general [37]: first, as contributions of higher powers in $\beta$ become numerically important when calculating the inclusive cross section, one has to match the resummed cross section to the full fixed order result. This is achieved by adding the latter, while subtracting all terms up to $\mathcal{O}\left(a_{s}\right)$ (modulo the common prefactor $\alpha_{s}^{2}$ ) of the expanded NNLL expression. In order to perform the matching on the $\mathrm{NNLO}_{\text {approx }}$ partonic cross section, one needs to subtract all terms up to $\mathcal{O}\left(a_{s}^{2}\right)$ of expanded resummation formula (denoted by NNLL(2)) and add back the approximated NNLO result [29].

If one chooses to resum in Mellin space, an inverse Mellin transformation is required which has to be done numerically. Due to omitted $1 / N$ contributions in the resummation formula (2.2), the matching onto the NNLO approximation will give a slightly different result compared to the NLO matching. ${ }^{4}$ The resummed partonic cross section is given by

$$
\hat{\sigma}_{i j \rightarrow \widetilde{g} \widetilde{g}}^{\mathrm{res}, \mathrm{NNLO}_{\text {approx }}}=\mathbf{M}^{-1}\left[\hat{\sigma}_{i j \rightarrow \widetilde{g} \widetilde{g}}^{\mathrm{NNLL,N}}-\hat{\sigma}_{i j \rightarrow \widetilde{g} \widetilde{g}}^{\mathrm{NNLL}(2), \mathrm{N}}\right]+\hat{\sigma}_{i j \rightarrow \widetilde{g} \widetilde{g}}^{\mathrm{NNLO}_{\text {approx }}} .
$$

The numerical Mellin inversion requires an analytic continuation of the resummed cross section to the complex plane. In our case, one simply assumes complex arguments of the logarithms. A more general discussion on that topic is provided in [38, 39], and a good routine is included in the program ANCONT [38]. Here, the Mellin inversion is obtained by

$$
f(\rho)=\frac{1}{\pi} \int_{0}^{\infty} d z \operatorname{Im}\left[e^{i \Phi} \rho^{-c(z)} \mathbf{M}[f](c(z))\right]
$$

where Mellin- $N$ is identified with $c(z)-1$, and $c(z)=c_{0}+z e^{i \Phi}$. As the contour integral around the singularity at $N=0$ is symmetric with respect to the $x$-axis, only the upper half is evaluated and multiplied by two. The path is chosen to be a truncated line with an angle $\Phi$ close to $\pi$. The parameter integral over $z$ is divided logarithmically into 20

\footnotetext{
${ }^{4}$ Here, one has to emphasize that the numerically inverted NNLL(2) cross section does not exactly correspond to threshold terms $\mathrm{NNLO}_{\mathrm{th}}$ in momentum space but comes close towards the threshold.
} 

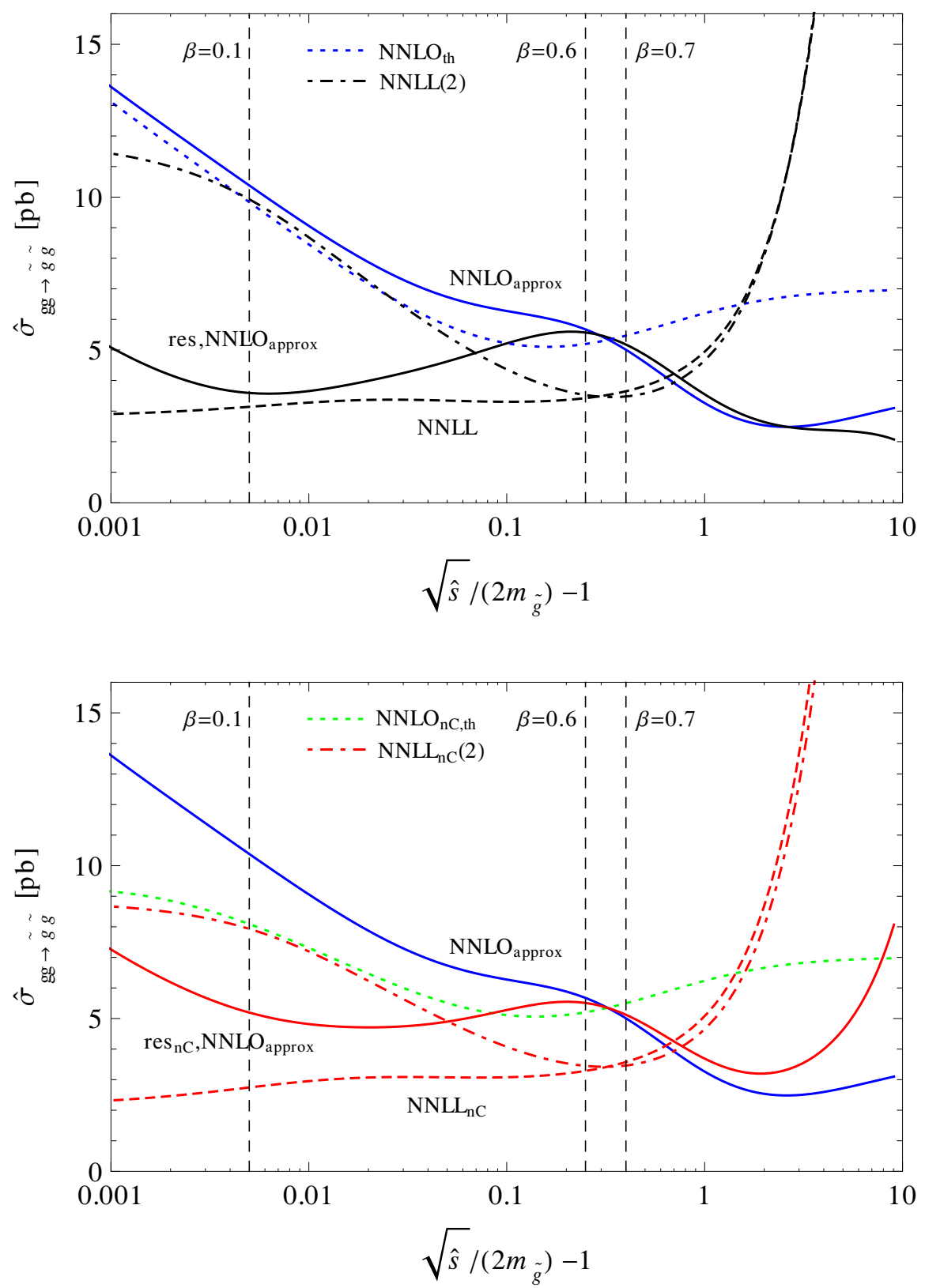

Figure 1. Partonic cross section for gluon fusion versus the energy above the production threshold normalized to the gluino pair mass. The renormalization and factorization scales have been set to $\mu=m_{\widetilde{g}}=800 \mathrm{GeV}$. We plot the NNLO approximation which is exact up to NLO, and the NNLO threshold limit $\mathrm{NNLO}_{\text {th }}$ which contains only threshold-enhanced contributions and NLO constants. Moreover, we show the NNLL resummed cross section and its expansion in $\alpha_{s}$ up to second order, NNLL(2), as well as the resummed partonic cross section matched onto the NNLO approximation. First panel: Coulomb corrections are included at fixed order in the resummation formula. Second panel: Coulomb corrections are neglected during resummation but kept in the NNLO approximation. 
pieces, where each segment is performed by the 32-point Gauss formula. We choose the starting point $c_{0}=1.4$ to the right of the singularity at $c(z)=1$, but left to the Landau pole singularity which is excluded from the integration contour according to the minimal prescription presented in ref. [19, 20].

In figure 1, we plot the resummed partonic cross section in the gluon fusion channel against the energy above the threshold normalized to the gluino pair mass $E /\left(2 m_{\widetilde{g}}\right)=$ $\sqrt{\hat{s}} /\left(2 m_{\widetilde{g}}\right)-1$ (solid black). The Coulomb corrections are included at fixed NNLO, and we set $\mu=m_{\tilde{g}}$. We also plot the NNLO approximation (solid blue) and the threshold approximation $\mathrm{NNLO}_{\text {th }}$ (dotted blue). Concerning the latter, all analytical input is given in ref. [13]. As a check of the numerical Mellin inversion, we further show the inverted expansion of the soft resummation NNLL(2) which comes close to $\mathrm{NNLO}_{\text {th }}$ for $\beta<0.5$. The inverted soft resummation NNLL merges with its $\mathcal{O}\left(\alpha_{s}^{2}\right)$ expansion away from the threshold. ${ }^{5}$ For low velocities $(\beta \ll 0.1)$, the numerical quality of the Mellin inversion decreases. As this systematic error affects both NNLL and NNLL(2), one may expect a cancellation in the difference to some extent. One the other hand, $\beta<0.1$ corresponds to an energy region $E<10 \mathrm{GeV}$ for $m_{\widetilde{g}} \approx 1 \mathrm{TeV}$ which is of minor importance for the evaluation of the total hadronic cross section. In the second panel, the resummation is done by omitting all Coulomb corrections (these are still included in the NNLO approximation). In figure 2, we compare the resummed cross section to the exact NLO result, where we also plot the theoretical error related to scale variation in the interval $\mu \in\left[m_{\widetilde{g}} / 2,2 m_{\widetilde{g}}\right]$.

As evident from the figures, the NNLL soft resummation significantly depletes the partonic cross section compared to the NNLO approximation and brings it down to the level of the NLO calculation for $\beta>0.1$. Close to the threshold, the resummed cross section is enhanced compared to fixed NLO but still well below the NNLO approximation. In the kinematical region $\beta \in[0.6,0.7]$, the resummed cross section, the NNLO approximation, and the threshold limit $\mathrm{NNLO}_{\text {th }}$ cross each other close by. For higher velocities $(\beta>0.7)$, threshold approximations become untrustworthy. In our analysis we set both terms in the square brackets of eq. (4.1) to zero if $\beta$ exceeds the value 0.64 . This choice gives rise to a smooth transition between the fixed-order and the resummed partonic cross section. Note that for the gluon fusion channel, the results are rather stable against a variation of the squark masses which enter at NLO. In constructing figure 1, we have assumed as example $m_{\widetilde{g}}=800 \mathrm{GeV}$ and a common squark mass of $640 \mathrm{GeV}$. Choosing squark masses above $1 \mathrm{TeV}$ will not change the conclusions drawn above. This is not true for the quark-antiquark annihilation channel. However, the hadronic production cross section is mainly driven by gluon fusion (about 99\%). Thus, we will not have a closer look on $q \bar{q}$-annihilation.

\section{Implementation of the momentum-space formalism}

Within the momentum-space formalism, no inversion is required and one can remove the operator $\mathbf{M}^{-1}$ in eq. (4.1). However, the $\omega$-integral over the potential function in eq. (3.2) requires an analytic continuation to negative values of $\eta$, where the integrand has to be

\footnotetext{
${ }^{5}$ At high velocities $(\beta>0.95)$, the two results drift apart again. However, in this region the factorization ansatz in eq. (2.2) is not valid and the resummation formula should not be used.
} 


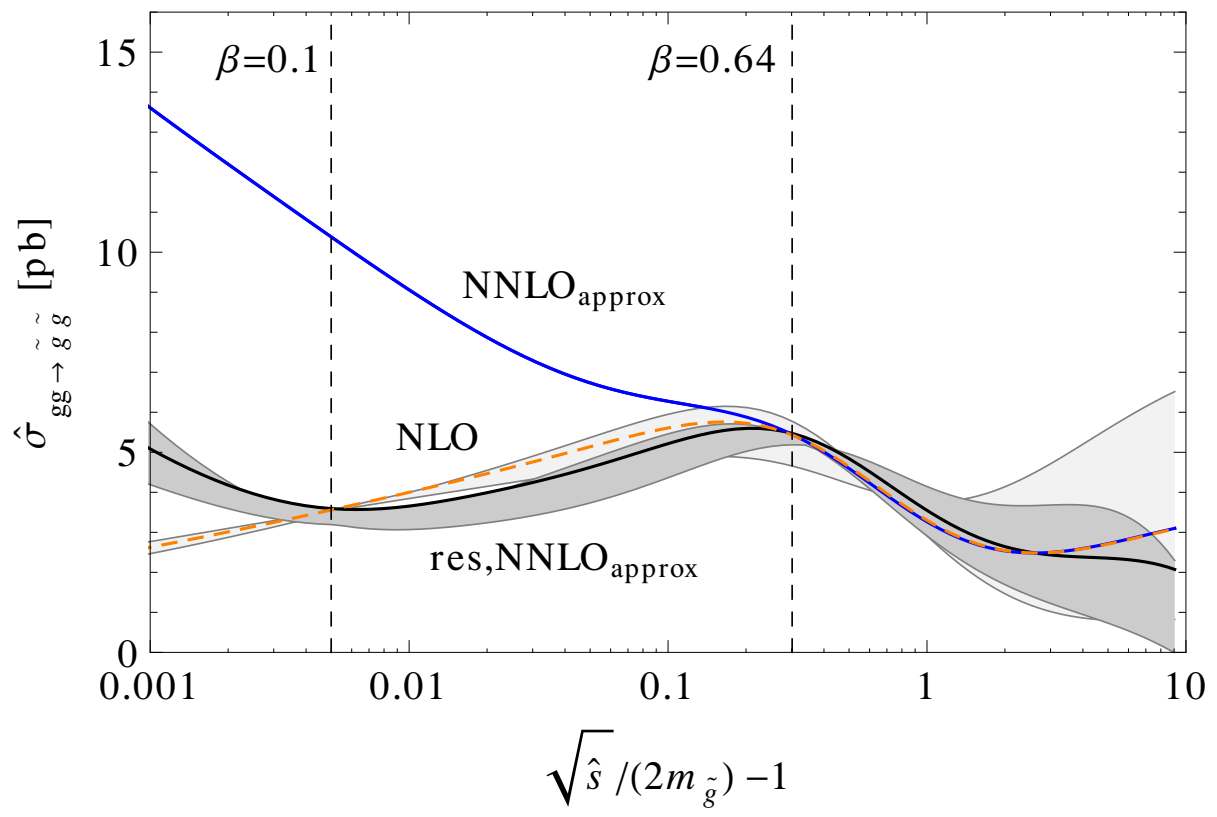

Figure 2. Comparison of the resummed partonic cross section to the exact NLO result and the NNLO approximation. The scale is varied within the interval $\mu \in\left[m_{\widetilde{g}} / 2,2 m_{\widetilde{g}}\right]$ around $\mu=m_{\widetilde{g}}=$ $800 \mathrm{GeV}$.

understood in a distributional sense such that the integral is convergent. For NNLL resummation of gluino pairs we need to integrate

$$
I(\omega)=\frac{1}{\omega}\left(\frac{\omega}{2 m_{\widetilde{g}}}\right)^{2 \eta} \frac{e^{-2 \gamma_{E} \eta}}{\Gamma(2 \eta)}\left(I^{(0)}(\omega)+16 \pi^{2} a_{s}^{2} I^{(0) S}(\omega)+I^{(1)}(\omega)\right),
$$

where

$$
\begin{aligned}
I^{(0)}(\omega)= & J_{\mathbf{I}}^{(0)}(E-\omega / 2)\left(1+4 a_{s}\left[C_{\mathbf{I}}\left(1+\hat{\psi}(2 \eta)+\ln \left(\frac{\mu_{s}}{2 m_{\widetilde{g}}}\right)\right)\right.\right. \\
& +\left(C_{i}+C_{j}\right)\left(\frac{\pi^{2}}{24}+\hat{\psi}(2 \eta)^{2}-\psi^{\prime}(2 \eta)+2 \hat{\psi}(2 \eta) \ln \left(\frac{\mu_{s}}{2 m_{\widetilde{g}}}\right)+\ln ^{2}\left(\frac{\mu_{s}}{2 m_{\widetilde{g}}}\right)\right) \\
& -\left\{C_{\mathbf{I}}+2\left(C_{i}+C_{j}\right)\left(\hat{\psi}(2 \eta)+\ln \left(\frac{\mu_{s}}{2 m_{\widetilde{g}}}\right)\right)\right\} \ln \left(\frac{\omega}{2 m_{\widetilde{g}}}\right) \\
& \left.\left.+\left(C_{i}+C_{j}\right) \ln ^{2}\left(\frac{\omega}{2 m_{\widetilde{g}}}\right)\right]\right) \\
I^{(0) S}(\omega)= & J_{\mathbf{I}}^{(0)}(E-\omega / 2) \ln \left(\frac{E-\omega / 2}{m_{\widetilde{g}}}\right) \\
I^{(1)}(\omega)= & J_{\mathbf{I}}^{(1)}(E-\omega / 2) .
\end{aligned}
$$

Here, we have defined $\hat{\psi}(x)=\gamma_{E}+\psi(x)$, where $\psi$ denotes the digamma function. Above the threshold, the integral runs from 0 to $2 E$. As outlined in ref. $[8,9]$ for instance, analytic 
continuation to negative values of $\eta$ can be achieved by a replacement of the integration kernel by a so-called star distribution, which contains subtraction terms that render the integral finite for $\eta>-1$. Explicitly, one applies

$$
\begin{aligned}
& \int_{0}^{2 E} d \omega f\left(E-\frac{\omega}{2}\right)\left[\frac{1}{\omega}\left(\frac{\omega}{2 m_{\tilde{g}}}\right)^{2 \eta}\right]_{*} \\
& =\int_{0}^{2 E} \frac{d \omega}{\omega}\left[f\left(E-\frac{\omega}{2}\right)-f(E)+\frac{\omega}{2} f^{\prime}(E)\right]\left(\frac{\omega}{2 m_{\widetilde{g}}}\right)^{2 \eta}+\left[\frac{f(E)}{2 \eta}-\frac{f^{\prime}(E) E}{2 \eta+1}\right]\left(\frac{E}{m_{\widetilde{g}}}\right)^{2 \eta}, \\
& \times \int_{0}^{2 E} d \omega f\left(E-\frac{\omega}{2}\right)\left[\frac{\ln \left(\frac{\omega}{2 m_{\tilde{g}}}\right)}{\omega}\left(\frac{\omega}{2 m_{\tilde{g}}}\right)^{2 \eta}\right]_{*} \\
& =\int_{0}^{2 E} \frac{d \omega}{\omega}\left[f\left(E-\frac{\omega}{2}\right)-f(E)+\frac{\omega}{2} f^{\prime}(E)\right] \ln \left(\frac{\omega}{2 m_{\tilde{g}}}\right)\left(\frac{\omega}{2 m_{\tilde{g}}}\right)^{2 \eta} \\
& +\left[\frac{f(E)}{2 \eta}\left(L_{E}-\frac{1}{2 \eta}\right)-\frac{f^{\prime}(E) E}{2 \eta+1}\left(L_{E}-\frac{1}{2 \eta+1}\right)\right]\left(\frac{E}{m_{\widetilde{g}}}\right)^{2 \eta}, \\
& \times \int_{0}^{2 E} d \omega f\left(E-\frac{\omega}{2}\right)\left[\frac{\ln ^{2}\left(\frac{\omega}{2 m_{\tilde{g}}}\right)}{\omega}\left(\frac{\omega}{2 m_{\tilde{g}}}\right)^{2 \eta}\right]_{*} \\
& =\int_{0}^{2 E} \frac{d \omega}{\omega}\left[f\left(E-\frac{\omega}{2}\right)-f(E)+\frac{\omega}{2} f^{\prime}(E)\right] \ln ^{2}\left(\frac{\omega}{2 m_{\widetilde{g}}}\right)\left(\frac{\omega}{2 m_{\widetilde{g}}}\right)^{2 \eta} \\
& +\left[\frac{f(E)}{2 \eta}\left(L_{E}^{2}-\frac{1}{\eta} L_{E}+\frac{1}{2 \eta^{2}}\right)-\frac{f^{\prime}(E) E}{2 \eta+1}\left(L_{E}^{2}-\frac{2}{2 \eta+1} L_{E}+\frac{2}{(2 \eta+1)^{2}}\right)\right]\left(\frac{E}{m_{\widetilde{g}}}\right)^{2 \eta},
\end{aligned}
$$

with $L_{E} \equiv \ln \left(E / m_{\tilde{g}}\right)$ and $f(E-\omega / 2)$ being a smooth test function on the interval $\omega \in[0,2 E]$. For positive $\eta$, one can drop the star-brackets and the above relations become simple identities. For negative $\eta$, diverging boundary terms at $\omega=0$ are removed by the star prescription. In deriving the fixed order expansion (3.16), we have assumed positive $\eta$ in order to perform the convolution over the LO potential function. We want to stress here that there are different methods for the analytic continuation. An alternative treatment, based on integration by parts, has been proposed in ref. [29]. However, due to the identity theorem for holomorphic functions, the continuation to $\eta<0$ is unique as the prescriptions agree for positive $\eta .{ }^{6} \mathrm{~A}$ different ansatz for analytic continuation is required for the non-Coulomb corrections. Here, one may apply

$$
\begin{aligned}
& \int_{0}^{2 E} d \omega J_{\mathbf{I}}^{(0)}\left(E-\frac{\omega}{2}\right) \ln \left(\frac{E-\omega / 2}{m_{\widetilde{g}}}\right)\left[\frac{1}{\omega}\left(\frac{\omega}{2 m_{\widetilde{g}}}\right)^{2 \eta}\right]_{*} \\
& =\int_{0}^{2 E} \frac{d \omega}{\omega}\left[J_{\mathbf{I}}^{(0)}\left(E-\frac{\omega}{2}\right)-J_{\mathbf{I}}^{(0)}(E)+\frac{\omega}{2} J_{\mathbf{I}}^{(0) \prime}(E)\right]\left(\frac{\omega}{2 m_{\widetilde{g}}}\right)^{2 \eta} \ln \left(\frac{E-\omega / 2}{m_{\widetilde{g}}}\right) \\
& +\left[\frac{J_{\mathbf{I}}^{(0)}(E)}{2 \eta}\left(L_{E}-\gamma_{E}-\psi(1+2 \eta)\right)-\frac{J_{\mathbf{I}}^{(0) \prime}(E) E}{2 \eta+1}\left(L_{E}-\gamma_{E}-\psi(2+2 \eta)\right)\right]\left(\frac{E}{m_{\widetilde{g}}}\right)^{2 \eta},
\end{aligned}
$$

\footnotetext{
${ }^{6}$ Here, I want to thank Pietro Falgari for clarifying discussions.
} 
where the terms in the last two lines have been evaluated for positive $\eta$. See also ref. [29] for a slightly different treatment. The latter relations can also be applied to the logarithm in eq. (3.9) if one includes the NLO corrections from the Coulomb potential at fixed order.

The poles at $\eta=-1 / 2$ and $\eta=-1$ in eqs. (5.6)-(5.9) are canceled by the overall factor $1 / \Gamma(2 \eta)$ in formula (5.1), see also the discussion in ref. [8,9]. The pole at $\eta=0$ is only canceled if there is no logarithm $\ln (\omega)$ contained in the integral. Otherwise, the prefactor $1 / \Gamma(2 \eta)$ is not sufficient to give a final result for $\eta \rightarrow 0$ as can be seen on the right-hand sides of eqs. (5.7) and (5.8). However, this does not cause any trouble. The limit $\eta \rightarrow 0$ corresponds to $\mu_{s} \rightarrow \mu_{f}$ which, using a running soft scale as stated above, is achieved for large velocities $(\beta \approx 1)$. However, the factorization ansatz in eq. (3.1) is not valid in that kinematical region. ${ }^{7}$ For instance, it is easy to see that some NLO terms in the soft function dominate the LO term, when $\eta$ approaches zero. Thus, one should not apply resummation for $\beta$ close to one. This will exclude the problematic case $\eta \rightarrow 0$.

Another complication arises due to the terms proportional to $f(E) L_{E}^{k} E^{2 \eta}$ with $k=$ $0,1,2$ in the formulas (5.6)-(5.8), and $J_{\mathbf{I}}^{(0)}(E) L_{E} E^{2 \eta}$ in eq. (5.9), which in most cases diverge for $E \rightarrow 0$. In our case, only the repulsive potential $J_{27}^{(0)}$ and its products with powers of $\ln (E)$ evaluate to zero at the threshold. For $\eta<-1 / 2$, the convolution with the parton luminosity is ill-defined, see also the equivalent discussion about top-quark pair production in ref. [29]. However, as outlined in the latter reference, the continuation to negative values of $\eta$ can be extended to the convolution over the hadronic cms energy producing a finite hadronic cross section.

At this point, we want to note that something seems to be different compared to the treatment in Mellin-space. Suppose that we neglect all kind of Coulomb corrections. It follows that the fixed order partonic cross section has to vanish near the threshold due to the overall factor $\beta$ in the born cross section. In Mellin space, this behavior is not spoiled by soft resummation. In the momentum-space approach in the absence of Coulomb corrections, the potential function reduces to the zeroth order in eq. (3.7) which is proportional to $\sqrt{E / m_{\widetilde{g}}} \approx \beta$. If this is multiplied by $E^{2 \eta}$, one finds a singular behavior for $E \rightarrow 0$ and $\eta<-1 / 4$ at the partonic level. Note that such a phenomenon does not occur in the discussion about deep inelastic scattering in ref. [8], simply because the momentum integral over $p^{2}$ runs from zero to $Q^{2}$ with $Q$ being the hard scale of the problem.

In any case one should state that resummation defines the partonic cross section as a distribution only irrespective if Coulomb terms are resummed or not. ${ }^{8}$ Therefore, there is no meaningful way to compare with the purely soft resummation in the Mellin-space formalism at the partonic level, where the threshold behavior mimics the fixed order in the absence of Coulomb terms, but adhoc assumptions have been made in order to deal with the Landau singularity.

We close this discussion by noting that threshold resummation in general suffers from ambiguities dealing with the question of how to treat the Landau pole on the one hand, or

\footnotetext{
${ }^{7}$ The same statement is also true for the corresponding ansatz of the Mellin-space approach in eq. (2.2), of course.

${ }^{8}$ Here, I want to thank Martin Beneke and Christian Schwinn for clarifying discussions.
} 
such related to the choice of scales and the matching procedure on the other. The explicit implementation of the momentum-space formalism is passed over to the experts on that approach.

\section{Hadronic cross section}

In this section, we examine the impact of the NNLL resummation onto the inclusive hadronic cross section for the examples $m_{\widetilde{g}}=800 \mathrm{GeV}$ and $m_{\widetilde{g}}=1 \mathrm{TeV}$. The current exclusion bounds suggest a minimum gluino mass of about $1 \mathrm{TeV}[1,2]$. However, we want to stress again that these results depend strongly on the chosen PDF set and the value of $\alpha_{s}\left(M_{Z}\right)$. The libraries CTEQ6.6 [40] and MSTW08 [41] used in ref. [1] lead to similar results of the hadronic production cross section. The more recent sets ABM11 [42] for instance differ from the latter at large parton momentum fraction $x$ which is of special importance for heavy (s)particle production. As discussed in ref. [13], the usage of ABM11 with $\alpha_{s}\left(M_{Z}\right)=0.1134 \pm 0.0011$ compared to MSTW08 featuring $\alpha_{s}\left(M_{Z}\right)=0.1171 \pm 0.0014$ reduces the hadronic production cross section by a factor of about two. Concerning the relative effect of the NNLL resummation compared to fixed NLO or $\mathrm{NNLO}_{\text {approx }}$, the actual choice of the PDF set will only play a minor role. In our analysis, we compare the widely used MSTW08 with ABM11 PDFs, where we always use NNLO sets also for the LO and NLO cross sections. We further assume a fixed ratio $m_{\widetilde{q}} / m_{\widetilde{g}}=4 / 5$ giving rise to the values $m_{\widetilde{q}}=640 \mathrm{GeV}$ and $m_{\widetilde{q}}=800 \mathrm{GeV}$. As mentioned above, this choice has also no significant impact on the result. A variation of the squark masses would affect the inclusive hadronic cross section at the percent level only.

The theoretical error due to higher order corrections is estimated by a variation of the scale $\mu=\mu_{f}=\mu_{h}$ in the range $\mu \in\left[m_{\tilde{g}} / 2,2 m_{\tilde{g}}\right]$. Within the momentum-space approach, one further has the possibility for a variation of the soft scale in order to account for the ambiguities within the soft resummation, as well as an independent variation of the hard scale. In principle, the latter is also possible in the Mellin-space approach. For illustration of the perturbative accuracy we will not include the PDF errors. Plots showing these can be found in ref. [13]. The convolution of the (resummed) partonic cross sections with the parton luminosity functions according to eq. (1.1) is performed by a VEGAS integration routine using LHAPDF grid files [43] for the PDF sets.

In the upper panel of figure 3 , we plot the fixed order inclusive cross section as a function of the hadronic cms energy $\sqrt{s}$ using ABM11 PDF sets. Explicit numbers for $\sqrt{s}=$ 7,8 , and $14 \mathrm{TeV}$ are given in table 1 . For $m_{\widetilde{g}}=800 \mathrm{GeV}$ and $\sqrt{s}=7 \mathrm{TeV}$, we observe the $K$-factors $K_{\mathrm{NLO}}=\sigma_{\mathrm{NLO}} / \sigma_{\mathrm{LO}}=1.55$ and $K_{\mathrm{NNLO}}=\sigma_{\mathrm{NNLO}_{\text {approx }}} / \sigma_{\mathrm{NLO}}=1.29$, at $14 \mathrm{TeV}$ we have $K_{\mathrm{NLO}}=1.50$ and $K_{\mathrm{NNLO}}=1.21$. For $m_{\widetilde{g}}=1 \mathrm{TeV}$ a similar picture emerges with $K_{\mathrm{NLO}}=1.63$ and $K_{\mathrm{NNLO}}=1.31$ for $\sqrt{s}=7 \mathrm{TeV}$, and $K_{\mathrm{NLO}}=1.48$ and $K_{\mathrm{NNLO}}=1.23$ at $14 \mathrm{TeV}$. In the lower panel of figure 3 , we compare the NNLO approximation to the NNLL resummation matched onto the latter. Explicit numbers are also given in table 1. In both examples, the $K$-factors $K_{\mathrm{NNLL}}=\sigma_{\text {res, } \mathrm{NNLO}_{\text {approx }}} / \sigma_{\mathrm{NLO}}$ are slightly below one over the whole range of hadronic cms energies. This is a direct consequence of the partonic result shown in figure 2 , where after convolution with the parton luminosities the corrections due 

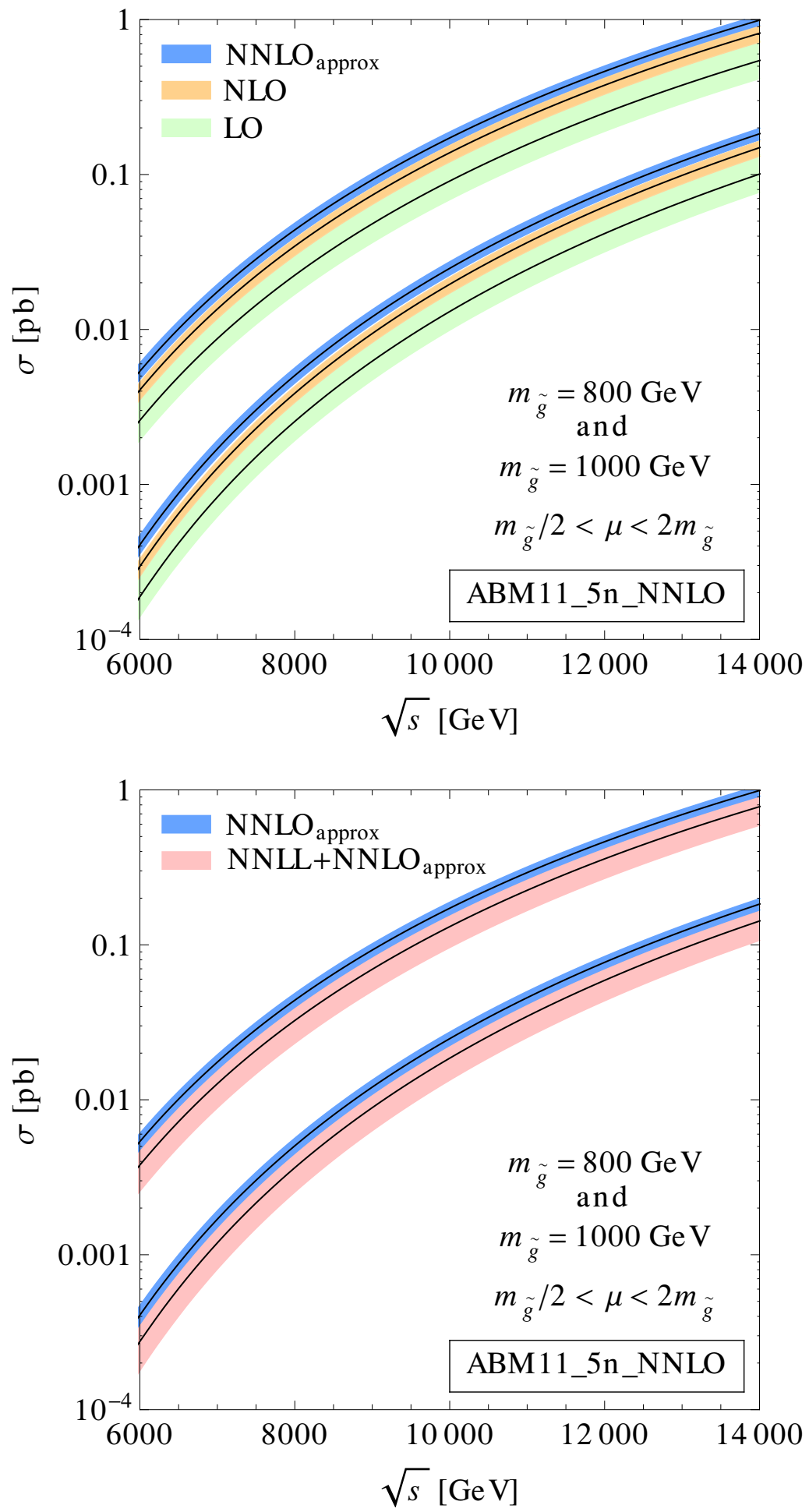

Figure 3. Inclusive hadronic cross section versus the squared cms energy. The error bars refer only to scale variation. Upper panel: fixed order for $m_{\widetilde{g}}=800 \mathrm{GeV}$ and $m_{\widetilde{g}}=1 \mathrm{TeV}$. Lower panel: NNLO approximation and soft NNLL resummation matched onto the latter. 


\begin{tabular}{|c|c|c|c|c|c|}
\hline $\begin{array}{c}m_{\widetilde{g}} \\
{[\mathrm{GeV}]}\end{array}$ & $\begin{array}{l}\sqrt{s} \\
{[\mathrm{TeV}]}\end{array}$ & $\begin{array}{l}\sigma^{\mathrm{LO}} \\
{[\mathrm{pb}]}\end{array}$ & $\begin{array}{l}\sigma^{\mathrm{NLO}} \\
{[\mathrm{pb}]}\end{array}$ & $\begin{array}{c}\sigma^{\sigma^{N N L O}} \text { approx } \\
{[\mathrm{pb}]}\end{array}$ & $\begin{array}{c}\sigma^{\text {res, }},{ }^{n L O} \text { approx } \\
{[\mathrm{pb}]}\end{array}$ \\
\hline \multicolumn{6}{|c|}{ MSTW 2008 NNLO } \\
\hline \multirow{3}{*}{800} & 7 & $0.0198\left(\begin{array}{l}0.0293 \\
0.0139\end{array}\right)$ & $0.0329\left(\begin{array}{l}0.0385 \\
0.0270\end{array}\right)$ & $0.0425\left(\begin{array}{l}0.0477 \\
0.0373\end{array}\right)$ & $0.0316\left(\begin{array}{l}0.0405 \\
0.0227\end{array}\right)$ \\
\hline & 8 & $0.0480\left(\begin{array}{l}0.0697 \\
0.0340\end{array}\right)$ & $0.0779\left(\begin{array}{l}0.0900 \\
0.0648\end{array}\right)$ & $0.0989\left(\begin{array}{l}0.1098 \\
0.0877\end{array}\right)$ & $0.0750\left(\begin{array}{l}0.0946 \\
0.0547\end{array}\right)$ \\
\hline & 14 & $0.9184\left(\begin{array}{l}1.2699 \\
0.6804\end{array}\right)$ & $1.3953\left(\begin{array}{l}1.5576 \\
1.2041\end{array}\right)$ & $1.6853\left(\begin{array}{l}1.8146 \\
1.5406\end{array}\right)$ & $1.3513\left(\begin{array}{l}1.6253 \\
1.0428\end{array}\right)$ \\
\hline \multirow{3}{*}{1000} & 7 & $0.0020\left(\begin{array}{l}0.0030 \\
0.0014\end{array}\right)$ & $0.0034\left(\begin{array}{l}0.0041 \\
0.0028\end{array}\right)$ & $0.0046\left(\begin{array}{l}0.0052 \\
0.0039\end{array}\right)$ & $0.0033\left(\begin{array}{l}0.0043 \\
0.0023\end{array}\right)$ \\
\hline & 8 & $0.0059\left(\begin{array}{l}0.0087 \\
0.0041\end{array}\right)$ & $0.0097\left(\begin{array}{l}0.0113 \\
0.0080\end{array}\right)$ & $0.0126\left(\begin{array}{l}0.0142 \\
0.0110\end{array}\right)$ & $0.0094\left(\begin{array}{l}0.0120 \\
0.0067\end{array}\right)$ \\
\hline & 14 & $0.1837\left(\begin{array}{l}0.2564 \\
0.1349\end{array}\right)$ & $0.2798\left(\begin{array}{l}0.3131 \\
0.2403\end{array}\right)$ & $0.3421\left(\begin{array}{l}0.3709 \\
0.3109\end{array}\right)$ & $0.2716\left(\begin{array}{l}0.3295 \\
0.2077\end{array}\right)$ \\
\hline \multicolumn{6}{|c|}{ ABM11 NNLO } \\
\hline \multirow{3}{*}{800} & 7 & $0.0087\left(\begin{array}{l}0.0119 \\
0.0065\end{array}\right)$ & $0.0135\left(\begin{array}{l}0.0151 \\
0.0117\end{array}\right)$ & $0.0175\left(\begin{array}{l}0.0195 \\
0.0156\end{array}\right)$ & $0.0127\left(\begin{array}{l}0.0165 \\
0.0088\end{array}\right)$ \\
\hline & 8 & $0.0224\left(\begin{array}{l}0.0304 \\
0.0168\end{array}\right)$ & $0.0345\left(\begin{array}{l}0.0385 \\
0.0299\end{array}\right)$ & $0.0439\left(\begin{array}{l}0.0486 \\
0.0394\end{array}\right)$ & $0.0325\left(\begin{array}{l}0.0416 \\
0.0231\end{array}\right)$ \\
\hline & 14 & $0.5439\left(\begin{array}{l}0.7289 \\
0.4148\end{array}\right)$ & $0.8160\left(\begin{array}{l}0.9031 \\
0.7150\end{array}\right)$ & $0.9908\left(\begin{array}{l}1.0674 \\
0.9085\end{array}\right)$ & $0.7790\left(\begin{array}{l}0.9476 \\
0.5897\end{array}\right)$ \\
\hline \multirow{3}{*}{1000} & 7 & $0.0008\left(\begin{array}{l}0.0011 \\
0.0006\end{array}\right)$ & $0.0013\left(\begin{array}{l}0.0014 \\
0.0011\end{array}\right)$ & $0.0017\left(\begin{array}{l}0.0019 \\
0.0015\end{array}\right)$ & $0.0012\left(\begin{array}{l}0.0016 \\
0.0008\end{array}\right)$ \\
\hline & 8 & $0.0025\left(\begin{array}{l}0.0035 \\
0.0019\end{array}\right)$ & $0.0039\left(\begin{array}{l}0.0043 \\
0.0034\end{array}\right)$ & $0.0050\left(\begin{array}{l}0.0057 \\
0.0045\end{array}\right)$ & $0.0036\left(\begin{array}{l}0.0048 \\
0.0025\end{array}\right)$ \\
\hline & 14 & $0.1006\left(\begin{array}{l}0.1346 \\
0.0768\end{array}\right)$ & $0.1490\left(\begin{array}{l}0.1641 \\
0.1311\end{array}\right)$ & $0.1831\left(\begin{array}{l}0.1986 \\
0.1674\end{array}\right)$ & $0.1424\left(\begin{array}{l}0.1750 \\
0.1067\end{array}\right)$ \\
\hline
\end{tabular}

Table 1. Hadronic cross section at $\sqrt{s}=7,8$, and $14 \mathrm{TeV}$ evaluated by using MSTW 2008 and ABM11 PDF sets. The ratio $m_{\widetilde{q}} / m_{\widetilde{g}}$ is kept fixed to the value $4 / 5$. The numbers in brackets correspond to the hadronic cross section evaluated at $\mu=m_{\tilde{g}} / 2$ (upper number) and $\mu=2 m_{\tilde{g}}$. PDF errors are not included.

to resummation arise mainly from the region $\beta \in[0.1,0.6]$. Similar observations are made by usage of MSTW 2008 PDFs which can be seen by inspection of table 1 . Here, one also notices that the central values exceed those due to ABM11 by about a factor of two as mentioned above. As discussed in ref. [13], this discrepancy is neither covered by the individual PDF errors, nor by the uncertainties due to scale variation which are given by the values in brackets.

Concerning the impact of scale variation, we find a rather large error for the NNLL resummation which even exceeds the uncertainty of the fixed NLO computation. This can also be understood from the partonic cross section shown in figure 2. In the region of relevance, the NNLL uncertainty is slightly larger then its NLO counterpart. At the same time the central values are lower, hence giving rise to a stronger relative impact of the scale variation. We conclude that for the case of heavy gluino pairs, soft resummation suggests that the fixed NLO description already gives a good theoretical estimate. An outstanding question is if this behavior is kept after Coulomb resummation which is likely to be answered within the momentum-space approach soon. 


\section{Conclusions}

In this article, we have discussed the effect of NNLL soft resummation on the inclusive cross section for gluino pair production at the LHC by the use of the Mellin-space resummation formalism. Here, we have used analytic results of ref. [13] and performed the Mellin inversion numerically by the use of standard methods. We have found that soft resummation compensates the enhancement of the NNLO soft logarithms compared to the fixed NLO result. This in turn suggests that the NLO computation already provides a good theoretical input for constructing exclusion limits at the LHC or, hopefully, to construct confidence regions for future evidences. At this point we want to stress again that the main source of uncertainty is related to the non-perturbative input when different PDF sets and initial values for $\alpha_{s}\left(M_{Z}\right)$ are applied. As discussed in ref. [13], the individual PDF errors are not sufficient to cover the resulting discrepancies.

We further calculated the hard function required for a joined soft and Coulomb resummation in the momentum-space approach. Here, we recaptured the relevant points concerning the convolution over the hadronic cms energy which are given in greater detail in the literature. Finally, we state that threshold resummation always suffers from ambiguities, no matter which approach is applied.

\section{Acknowledgments}

I would like to thank Ulrich Langenfeld and Sven-Olaf Moch for good advice and also for providing me with useful pieces of code. I further wish to thank Martin Beneke, Pietro Falgari, and Christian Schwinn for very useful discussions. This work has been supported in part by the Deutsche Forschungsgemeinschaft in Sonderforschungsbereich/Transregio 9 and by the European Commission through contract PITN-GA-2010-264564 (LHCPhenoNet).

\section{A Matching to the NNLO approximation}

In this appendix, we give the relevant part of the NNLO hard function which may be used for the matching onto the NNLO approximation. The specific choice given here sets all NNLO constants to zero within the resummation formula (3.2). For the generic scale choice $\mu_{s}=k_{s} m_{\widetilde{g}} \beta^{2}$, we obtain

$$
\begin{aligned}
& H_{i j, \mathbf{I}}^{(2) \operatorname{match}}=-C_{1, \mathbf{I}}^{i j}\left(48 C_{\mathbf{I}}(1-\ln (2))+2\left(C_{i}+C_{j}\right)\left(64-96 \ln (2)+72 \ln ^{2}(2)-\frac{11}{3} \pi^{2}\right)\right) \\
& +144 C_{\mathbf{I}}^{2}(1-\ln (2))+2\left(C_{i}+C_{j}\right) C_{\mathbf{I}}\left(384-480 \ln (2)+216 \ln ^{2}(2)+11 \pi^{2} \ln (2)-22 \pi^{2}\right) \\
& +2\left(C_{i}+C_{j}\right)^{2}\left(512-768 \ln (2)+576 \ln ^{2}(2)+44 \pi^{2} \ln (2)-33 \pi^{2}-\frac{176}{3} \pi^{2}+\frac{121}{72} \pi^{4}\right) \\
& +L_{\mu}\left\{-C_{1, \mathbf{I}}^{i j}\left(8 C_{\mathbf{I}}+16\left(C_{i}+C_{j}\right)(2-3 \ln (2))\right)-4 C_{\mathbf{I}}\left(33-2 n_{l}-3 \kappa_{i j}\right)(1-\ln (2))\right. \\
& +24 C_{\mathbf{I}}^{2}-\left(C_{i}+C_{j}\right)\left(\frac{1592}{3}-\frac{1}{3} C_{\mathbf{I}}\left(13 \pi^{2}+144 \ln (2)(1-2 \ln (2))\right)+\frac{n_{l}}{9}(-272+408 \ln (2)\right.
\end{aligned}
$$




$$
\begin{aligned}
& \left.-216 \ln ^{2}(2)+11 \pi^{2}\right)+\kappa_{i j}\left(-32+48 \ln (2)-36 \ln ^{2}(2)+\frac{11}{6} \pi^{2}\right)-268 \ln (2)-528 \ln (2) \\
& \left.+396 \ln ^{2}(2)+12 \pi^{2} \ln (2)-\frac{169}{6} \pi^{2}\right)-\frac{2}{3}\left(C_{i}+C_{j}\right)^{2}\left(768-960 \ln (2)+1008 \ln ^{2}(2)\right. \\
& \left.\left.-432 \ln (2)^{3}+61 \pi^{2} \ln (2)-48 \pi^{2}-336 \zeta(3)\right)+\gamma_{i}^{\phi(1)}+\gamma_{j}^{\phi(1)}+\gamma_{i j, \mathbf{I}}^{V(1)}\right\}+L_{\mu}^{2}\left\{-4 C_{1, \mathbf{I}}^{i j}\left(C_{i}+C_{j}\right)\right. \\
& -C_{\mathbf{I}}\left(11-\frac{2}{3} n_{l}-2 \kappa_{i j}\right)+2 C_{\mathbf{I}}^{2}+\left(C_{i}+C_{j}\right)\left(-\frac{199}{3}+4 C_{\mathbf{I}}(3-2 \ln (2))+\frac{2}{9} n_{l}(17-18 \ln (2))\right. \\
& \left.\left.+4 \kappa_{i j}(2-3 \ln (2))+66 \ln (2)+\pi^{2}\right)+\left(C_{i}+C_{j}\right)^{2}\left(-32+64 \ln (2)-24 \ln ^{2}(2)+\frac{13}{6} \pi^{2}\right)\right\} \\
& +L_{\mu}^{3}\left\{\left(C_{i}+C_{j}\right)\left(-\frac{11}{3}+2 C_{\mathbf{I}}+\frac{2}{9} n_{l}+\kappa_{i j}\right)-4\left(C_{i}+C_{j}\right)^{2} \ln (2)\right\}+\frac{1}{2}\left(C_{i}+C_{j}\right)^{2} L_{\mu}^{4}, \\
& +\ln \left(k_{s}\right)\left\{-\left(\gamma_{i}^{\phi(1)}+\gamma_{j}^{\phi(1)}\right)-\gamma_{i j, \mathbf{I}}^{V(1)}+4 C_{\mathbf{I}}\left(33-2 n_{l}\right)(1-\ln (2))+24 C_{\mathbf{I}}^{2}(1-\ln (2))\right. \\
& +\frac{1}{2}\left(C_{i}+C_{j}\right)\left(\frac{3184}{3}+\frac{2}{3} C_{\mathbf{I}}\left(672-1008 \ln (2)+648 \ln (2)^{2}-35 \pi^{2}\right)-\frac{2}{9} n_{l}(272-408 \ln (2)\right. \\
& \left.\left.+216 \ln (2)^{2}-11 \pi^{2}\right)-1592 \ln (2)+792 \ln (2)^{2}+24 \pi^{2} \ln (2)-\frac{169}{3} \pi^{2}\right) \\
& \left.+\frac{2}{3}\left(C_{i}+C_{j}\right)^{2}\left(1152-1728 \ln (2)+1296 \ln (2)^{2}-648 \ln (2)^{3}+105 \pi^{2} \ln (2)-70 \pi^{2}-336 \zeta_{3}\right)\right\} \\
& +\ln ^{2}\left(k_{s}\right)\left\{2 C_{\mathbf{I}}^{2}+C_{\mathbf{I}}\left(11-\frac{2}{3} n_{l}\right)+\frac{1}{2}\left(C_{i}+C_{j}\right)\left(\frac{398}{3}+8 C_{\mathbf{I}}(7-9 \ln (2))-n_{l}\left(\frac{68}{9}-8 \ln (2)\right)\right.\right. \\
& \left.\left.-132 \ln (2)-2 \pi^{2}\right)+\frac{1}{6}\left(C_{i}+C_{j}\right)^{2}\left(576-864 \ln (2)+648 \ln (2)^{2}-35 \pi^{2}\right)\right\} \\
& +\ln ^{3}\left(k_{s}\right)\left\{\frac{1}{2}\left(C_{i}+C_{j}\right)\left(\frac{22}{3}+4 C_{\mathbf{I}}-\frac{4}{9} n_{l}\right)+4\left(C_{i}+C_{j}\right)^{2}(2-3 \ln (2))\right\}+\ln ^{4}\left(k_{s}\right) \frac{1}{2}\left(C_{i}+C_{j}\right)^{2},
\end{aligned}
$$

where $\kappa_{g g}=0$ and $\kappa_{q \bar{q}}=-2 \beta_{0}+6 C_{F}$. The relative correction to the NLO hard function is about a few percent only.

Open Access. This article is distributed under the terms of the Creative Commons Attribution License which permits any use, distribution and reproduction in any medium, provided the original author(s) and source are credited.

\section{References}

[1] ATLAS collaboration, Search for squarks and gluinos with the ATLAS detector in final states with jets and missing transverse momentum using $4.7 \mathrm{fb}^{-1}$ of $\sqrt{s}=7 \mathrm{TeV}$ proton-proton collision data, Phys. Rev. D 87 (2013) 012008 [arXiv:1208.0949] [INSPIRE].

[2] CMS collaboration, Inclusive search for supersymmetry using the razor variables in $p p$ collisions at $\sqrt{s}=7 \mathrm{TeV}$, arXiv:1212.6961 [INSPIRE].

[3] W. Beenakker, R. Hopker, M. Spira and P. Zerwas, Squark and gluino production at hadron colliders, Nucl. Phys. B 492 (1997) 51 [hep-ph/9610490] [INSPIRE]. 
[4] W. Beenakker, R. Hopker and M. Spira, PROSPINO: a program for the production of supersymmetric particles in next-to-leading order QCD, hep-ph/9611232 [INSPIRE].

[5] G.F. Sterman, Summation of large corrections to short distance hadronic cross-sections, Nucl. Phys. B 281 (1987) 310 [inSPIRE].

[6] S. Catani and L. Trentadue, Resummation of the QCD perturbative series for hard processes, Nucl. Phys. B 327 (1989) 323 [inSPIRE].

[7] T. Becher and M. Neubert, Threshold resummation in momentum space from effective field theory, Phys. Rev. Lett. 97 (2006) 082001 [hep-ph/0605050] [inSPIRE].

[8] T. Becher, M. Neubert and B.D. Pecjak, Factorization and momentum-space resummation in deep-inelastic scattering, JHEP 01 (2007) 076 [hep-ph/0607228] [INSPIRE].

[9] M. Beneke, P. Falgari and C. Schwinn, Threshold resummation for pair production of coloured heavy (s)particles at hadron colliders, Nucl. Phys. B 842 (2011) 414 [arXiv: 1007.5414] [INSPIRE].

[10] P. Falgari, C. Schwinn and C. Wever, NLL soft and Coulomb resummation for squark and gluino production at the LHC, JHEP 06 (2012) 052 [arXiv:1202.2260] [INSPIRE].

[11] A. Kulesza and L. Motyka, Soft gluon resummation for the production of gluino-gluino and squark-antisquark pairs at the LHC, Phys. Rev. D 80 (2009) 095004 [arXiv: 0905.4749] [INSPIRE].

[12] W. Beenakker et al., Soft-gluon resummation for squark and gluino hadroproduction, JHEP 12 (2009) 041 [arXiv: 0909.4418] [INSPIRE].

[13] U. Langenfeld, S.-O. Moch and T. Pfoh, QCD threshold corrections for gluino pair production at hadron colliders, JHEP 11 (2012) 070 [arXiv: 1208.4281] [INSPIRE].

[14] K. Hagiwara and H. Yokoya, Bound-state effects on gluino-pair production at hadron colliders, JHEP 10 (2009) 049 [arXiv:0909.3204] [INSPIRE].

[15] M.R. Kauth, J.H. Kuhn, P. Marquard and M. Steinhauser, Gluino pair production at the LHC: the threshold, Nucl. Phys. B 857 (2012) 28 [arXiv:1108.0361] [INSPIRE].

[16] P. Falgari, C. Schwinn and C. Wever, Finite-width effects on threshold corrections to squark and gluino production, JHEP 01 (2013) 085 [arXiv:1211.3408] [INSPIRE].

[17] S. Catani and L. Trentadue, Comment on QCD exponentiation at large $x$, Nucl. Phys. B 353 (1991) 183 [INSPIRE].

[18] H. Contopanagos, E. Laenen and G.F. Sterman, Sudakov factorization and resummation, Nucl. Phys. B 484 (1997) 303 [hep-ph/9604313] [INSPIRE].

[19] S. Catani, M.L. Mangano, P. Nason and L. Trentadue, The top cross-section in hadronic collisions, Phys. Lett. B 378 (1996) 329 [hep-ph/9602208] [INSPIRE].

[20] S. Catani, M.L. Mangano, P. Nason and L. Trentadue, The resummation of soft gluons in hadronic collisions, Nucl. Phys. B 478 (1996) 273 [hep-ph/9604351] [INSPIRE].

[21] N. Kidonakis and G.F. Sterman, Resummation for QCD hard scattering, Nucl. Phys. B 505 (1997) 321 [hep-ph/9705234] [INSPIRE].

[22] S. Moch, J. Vermaseren and A. Vogt, Higher-order corrections in threshold resummation, Nucl. Phys. B 726 (2005) 317 [hep-ph/0506288] [INSPIRE]. 
[23] H. Kawamura, N. Lo Presti, S. Moch and A. Vogt, On the next-to-next-to-leading order QCD corrections to heavy-quark production in deep-inelastic scattering, Nucl. Phys. B 864 (2012) 399 [arXiv: 1205.5727] [INSPIRE].

[24] M. Beneke, M. Czakon, P. Falgari, A. Mitov and C. Schwinn, Threshold expansion of the $g g(q \bar{q}) \rightarrow Q \bar{Q}+X$ cross section at $O\left(\alpha_{s}^{4}\right)$, Phys. Lett. B $690(2010) 483$ [arXiv:0911.5166] [INSPIRE].

[25] A. Czarnecki and K. Melnikov, Two loop QCD corrections to the heavy quark pair production cross-section in $e^{+} e^{-}$annihilation near the threshold, Phys. Rev. Lett. 80 (1998) 2531 [hep-ph/9712222] [INSPIRE].

[26] M. Beneke, A. Signer and V.A. Smirnov, Top quark production near threshold and the top quark mass, Phys. Lett. B 454 (1999) 137 [hep-ph/9903260] [InSPIRE].

[27] A. Czarnecki and K. Melnikov, Top quark threshold production at a $\gamma \gamma$ collider at next-to-next-to-leading order, Phys. Rev. D 65 (2002) 051501 [hep-ph/0108233] [INSPIRE].

[28] A. Pineda and A. Signer, Heavy quark pair production near threshold with potential non-relativistic QCD, Nucl. Phys. B 762 (2007) 67 [hep-ph/0607239] [INSPIRE].

[29] M. Beneke, P. Falgari, S. Klein and C. Schwinn, Hadronic top-quark pair production with NNLL threshold resummation, Nucl. Phys. B 855 (2012) 695 [arXiv:1109.1536] [InSPIRE].

[30] T. Becher, M. Neubert and G. Xu, Dynamical threshold enhancement and resummation in Drell-Yan production, JHEP 07 (2008) 030 [arXiv: 0710.0680] [INSPIRE].

[31] S. Moch and P. Uwer, Theoretical status and prospects for top-quark pair production at hadron colliders, Phys. Rev. D 78 (2008) 034003 [arXiv:0804.1476] [INSPIRE].

[32] T. Becher and M. Neubert, On the structure of infrared singularities of gauge-theory amplitudes, JHEP 06 (2009) 081 [arXiv: 0903.1126] [INSPIRE].

[33] M. Beneke, P. Falgari and C. Schwinn, Soft radiation in heavy-particle pair production: all-order colour structure and two-loop anomalous dimension, Nucl. Phys. B 828 (2010) 69 [arXiv:0907.1443] [INSPIRE].

[34] M. Bonvini, S. Forte, M. Ghezzi and G. Ridolfi, The scale of soft resummation in SCET vs perturbative $Q C D$, arXiv:1301.4502 [INSPIRE].

[35] V.S. Fadin, V.A. Khoze and T. Sjöstrand, On the threshold behavior of heavy top production, Z. Phys. C 48 (1990) 613 [inSPIRE].

[36] M. Beneke, Y. Kiyo and K. Schuller, Third-order Coulomb corrections to the S-wave Green function, energy levels and wave functions at the origin, Nucl. Phys. B 714 (2005) 67 [hep-ph/0501289] [INSPIRE].

[37] R. Bonciani, S. Catani, M.L. Mangano and P. Nason, NLL resummation of the heavy quark hadroproduction cross-section, Nucl. Phys. B 529 (1998) 424 [Erratum ibid. B 803 (2008) 234] [hep-ph/9801375] [INSPIRE].

[38] J. Blumlein, Analytic continuation of Mellin transforms up to two loop order, Comput. Phys. Commun. 133 (2000) 76 [hep-ph/0003100] [INSPIRE].

[39] J. Blumlein and S. Kurth, Harmonic sums and Mellin transforms up to two loop order, Phys. Rev. D 60 (1999) 014018 [hep-ph/9810241] [INSPIRE].

[40] P.M. Nadolsky et al., Implications of CTEQ global analysis for collider observables, Phys. Rev. D 78 (2008) 013004 [arXiv:0802.0007] [INSPIRE]. 
[41] A. Martin, W. Stirling, R. Thorne and G. Watt, Parton distributions for the LHC, Eur. Phys. J. C 63 (2009) 189 [arXiv:0901.0002] [InSPIRE].

[42] S. Alekhin, J. Blumlein and S. Moch, Parton distribution functions and benchmark cross sections at NNLO, Phys. Rev. D 86 (2012) 054009 [arXiv:1202.2281] [INSPIRE].

[43] M. Whalley, D. Bourilkov and R. Group, The Les Houches accord PDFs (LHAPDF) and LHAGLUE, hep-ph/0508110 [INSPIRE]. 